\title{
NON-ALCOHOLIC FATTY LIVER DISEASE IN THE CONTEXT OF ALTERED GUT MICROBIOTA
}

DOI: 10.36740/WLek202104137

\author{
Tetyana V. Koval, Ivan V. Chopey, Mykhaylo M. Hechko, Artur V. Kurakh \\ STATE HIGHER EDUCATIONAL INSTITUTION “UZHHOROD NATIONAL UNIVERSITY”, UZHHOROD, UKRAINE
}

\begin{abstract}
The aim: To analyze the relationship between non-alcoholic fatty liver disease and changes in the gut microbiota.

Materials and methods: The publications of domestic and foreign editions in the databases of the United European Gastroenterology (UEG) Journal, PubMed, MEDLINE, Web of Science were processed and analyzed.

Conclusions: In recent years, non-alcoholic fatty liver disease was placed among the important diseases in gastroenterology. During this time, more and more data appear on the link between changes in the human intestinal microbiome and the development of metabolic diseases, including NAFLD. Contemporary research has indeed found evidence of such a relationship. Thus, some strains of microorganisms have been identified in more detail, which directly or indirectly affect the development or course of the above-mentioned disease. For a better understanding of the strategies for the treatment of pathologies, it is necessary to delve into the study of etiological factors, therefore, NAFLC cannot be considered a pathology that has been sufficiently studied. Indeed, recent data indicate that the development and severity of the course of the disease are not always associated with the physiological processes already known to us.
\end{abstract}

KEY WORDS: Non-alcoholic fatty liver disease, intestinal microbiota, human microbiome

\section{INTRODUCTION}

Currently, non-alcoholic fatty liver disease (NAFLD) is the leading cause of chronic liver disease worldwide, even in developed countries, and the number of patients is constantly growing. The worldwide prevalence of NAFLC is reported to be $24 \%$ [1]. NAFLD is very common in South America and the Middle East, followed by Asia, the United States and Europe (31\%, 32\%, 27\%, 24\% and 23\%, respectively), while there are fewer NAFLD patients in Africa (14\%). This pathology often has similar symptoms with other metabolic disorders, including type 2 diabetes and obesity. Recent studies have highlighted the role of gut microbiota composition in the pathophysiology of many diseases. With regard to NAFLD, experiments carried out using gut microbiota transplantation in animal models that did not have a tendency for NAFLD, showed that the development of fatty liver disease is associated with intestinal bacteria. Moreover, in patients with NAFLD, changes in the composition of the intestinal microbiota were observed.

\section{THE AIM}

Analyze the relationship between non-alcoholic fatty liver disease and changes in the intestinal microbiota based on the studies and works already described.

\section{MATERIALS AND METHODS}

To achieve this goal, publications of domestic and foreign publications found by stated keywords "Non-alcoholic liver disease", "changes in the intestinal microbiota", "overweight", "obesity" in the databases of the United European Gastroenterology (UEG) Journal, PubMed, MEDLINE, Web of Science were processed and analyzed.

\section{REVIEW AND DISCUSSION}

The microbiome of the gastrointestinal tract is represented by different bacteria, both quantity and quality. Their highest level and diversity are presented in the colon. Dominant groups of bacteria were presented by Firmicutes, Proteobacteria, Actinobacteria and Bacteroidetes. High levels of the latter correlated with higher individual susceptibility to pathologic conditions, including obesity. [2] However, to talk about the similarities in microbiota compositions at the population level is difficult and incorrect. After all, even identical twins have different microbiomes. [3] It is known that in the large intestine many bacteria coexist, including such important for its proper functioning bacteria as Escherichia coli (E. coli), Campylobacter jejuni, Salmonella enterica, Vibrio cholerae and Bacteroides fragilis, usually in very low quantities $(<0,1 \%$ intestinal microbiome). $[3,4]$ The microbiome differs in the same parts of the intestine, 
depending on its proximity to the intestinal mucosa. Thus, Bacteroides, Streptococcus, Bifdobacterium, Enterobacteriaceae, Enterococcus, Clostridium, Ruminococcus and Lactobacillus inhabit the parts that are farther from the mucosa, while Clostridium, Lactobacillus, Enterococcus and Akkermansia are located closer to its surface. [5] The composition of the microbiome is influenced by many external factors, such as diet, birth method, geographical location, genetics, etc. [6,7] The composition of the microbiome is unique to each person, but changes may occur during a lifetime and can affect the immune, metabolic and phenotypic processes of the host. [8] As stated by such authors as Kovacs and others, we it can be stated that genetics play a key role in the formation of the intestinal microbiome, as shown by studies on mice. [9] However, human subject research provide different results and so, even in genetically related individuals the structure of the intestinal microbiota didn 't have much similarities. $[10,11]$ Various manipulations that affect the change of intestinal microbiota lead to worsening or improvement of the course of a disease, and sometimes - to development of new diseases in a person. It is believed that the the link between the biological processes of the intestinal microbiota and the development of liver diseases is observed from the moment of fetal development, because the formation of the liver begins directly from the foregut. This link remains after birth, as the liver receives $75 \%$ of the blood flow from the intestinal portal. [12] The connection between the human microbiome and the development of NAFLD was first proposed in 1980, when patients with concomitant NAFLD underwent surgical bypass of the intestine, followed by the prescription of antibacterial drugs. Antibacterial therapy improved the course of NAFLD . [13]

Different studies on humans and animals, clearly outlined the connection between dysbacteriosis and NAFLD $[14,15]$, as well as the severity of the latter. [16] According to Spencer et al. it was found that along with the decrease in choline levels, the accumulation of fat in the liver depended on the level of Erysipelotrichia and Gammaproteobacteria in the intestine. Thus, according to these data, a higher level of Erysipelotrichia was observed in patients with a higher level of NAFLD, and a higher level of Gammaproteobacteria - on the contrary, was found in patients with a low NAFLD development. Comparisons were made between the two groups - underweight and obese patients. It was found that patients with NAFLD had an increase in the level of gram-negative bacteria (Bacteroidetes up to 20\%) and a decrease in the level of Firmicutes (SCFA-producing Lachnospiraceae, Lactobacillaceae and 7 $\alpha$-dehydroxylating Ruminococcaceae) to $24 \%$ compared to patients without obesity. However, in patients with NAFLD, there was an increase in the number of opportunistic pathogenic microorganisms that produce lipopolysaccharides, compared to the other group of patients. [17]

In 2017, new data emerged stating that even different forms of nonalcoholic fatty hepatosis are accompanied by different balance ratios of the intestinal microbiota. Thus, according to the Loomba et al, in patients with mild to moderate severity forms of NAFLD, a higher level of Firmicutes was observed compared to patients with severe fibrosis. In regards to Proteobacteria the situation was different - higher levels were found in patients with severe forms of fibrosis. In turn, Eubacterium was observed in higher numbers in patients with mild forms of fibrosis, while elevated levels of E. coli were detected in later stages of NAFLD, however, in this group of patients there was a decreased level of Ruminococcus obeum and E. Rectale [18] There was also other evidence of a relationship between intestinal microbiota imbalance and the development of NAFLD. Studies involving other classes of the intestinal microbiota, such as Allisonella and Parabacteroides, as well as Anaerosporobacter and Faecalibacterium, have shown that the former were more common in patients with a concomitant diagnosis of NAFLD than the other three [19].

According to Mouzaki et al. lower levels of Bacteroidetes were also observed in patients with nonalcoholic hepatosis, compared to patients without fibrosis. Moreover, according to these data, significant differences in the composition of the intestinal microbiota between patients with mild fibrosis and healthy patients were not found. [14]

Despite data on the influence of genetic factors on the composition of the hosts 'intestinal microbiota, it is known that changes in the relation of different species of bacteria also depend on lifestyle, including malnutrition, urbanization, reduced physical activity, worsened living conditions, uncontrolled use of antibiotics and improved hygiene. All of these factors affected the composition of our microbiota, as well as the emergence of so-called diseases of modern civilization, which contributes to changes in the microbiota and morbidity. [20] Diet is considered to be one of the most powerful aspects of microbial communities [21,22]. Studies have shown, that such changes in diet, like inclusion of high levels of sugar and fat, deeply affected the gut microbiota, with changes in the relation of Firmicutes to Bacteroidetes and an abrupt decrease in bacterial variety $[23,24]$

Recent data show that the appointment of antibacterial drugs, as the main method of treatment of many diseases today, leads to a significant reduction of important for the proper functioning of the body, microorganisms, and sometimes their disappearance from the patient's microbiome. [25] Instead, the literature describes data on the positive effects of prescribing antibacterial drugs, in particular in the treatment of NAFLD. Models were described, in which treatment with antibacterial drugs lasting 6 months, mainly the subsequent use of norfloxacin and neomycin, led to a decrease in bacterial growth of the microbiota in the intestinal lumen, which improved the course of NAFLD, as well as improved liver function in cirrhosis. [26] According to Gangarapu V et al., administration of rifaximin may reduce circulating endotoxin (LPS) and alanine aminotransferase levels in patients with NAFLD. [30] Back in 2008, Wu W showed that experimental oral administration of cidomycin increased the rate of transit through the small intestine and decreased the serum alanine aminotransferase, aspartate aminotransferase and 
tumor necrosis factor- $\alpha$ levels in rats with NASH, indicating the cydamicins potential for lowering NASH activity by changing the gut microbiome. [27]

However, recent reports show an increasing trend towards antibiotic resistance, so the widespread use of antibiotics is currently accompanied by a highly negative health response.

In recent years, there have been reports of a positive effect from intensive growth of some microorganisms on human health, namely, it was found that Akkermansia muciniphila is involved in the destruction of mucin, which, in turn, is positively correlated with weight loss and reduction of NAFLD risk. [28]

According to the latest EASL / EASD / EASO recommendations, pharmacological treatment of NAFLD begins only when liver fibrosis is confirmed. In other cases, non-pharmacologic methods are use, which mainly consist of lifestyle changes. However, the recommendations do not include any treatment aimed at correcting the composition of the intestinal microbiota, as this issue is not sufficiently studied.

Despite all the factors, at the moment diet remains one of the universal methods of influencing the composition of the microbiota. It is known that the entire human microbiome is actively involved in digestion. Its main role is to breakdown and absorb substances from food. Substances that penetrate the epithelial barrier, affect even the most remote cells of the body, using the bloodstream as a method of transportation. [29] This is supported by descriptions of L-carnitine metabolism, which is contained mainly in red meat. Gut microorganisms and the liver convert it into trimethylanin $\mathrm{N}$-oxide, which leads to the development of atherosclerosis. Thus, according to RA Koeth, in vegetarians the composition of the microbiota contributes to significantly less formation of trimethylanin $\mathrm{N}$-oxide compared to those who include meat in the diet. [30]

\section{CONCLUSIONS}

Thus, the intestinal microbiota may be associated with detrimental effects on the liver, in particular, the development of NAFLD, and changing the composition of the intestinal microbiota through diet may be an effective strategy to improve the course of liver disease. The main goals of steatohepatitis treatment include the reduction of its stage, lowering insulin resistance, prevention of steatosis progression and reduction of oxidative stress. The cornerstone of NAFLD treatment, of course, is lifestyle changes. At the same time, adherence to low calorie diet and reduction of consumption of foods and beverages with high fructose content, along with weight loss and reduction of liver steatosis, may quickly change the intestinal microbiome. The purpose of diet therapy is a gradual decrease in body weight by $1.5-2 \mathrm{~kg}$ per month (up to $7 \%$ or more) - this may reverse hepatic steatosis. In NASH, however, weight loss should be $\geq 10 \%$ of baseline.

Based on the above, there is an urgent need for further research to identify the link between the intestinal microbiota and the development and progression of NAFLD.

\section{REFERENCES}

1. Younossi Z.M., Koenig A.B., Abdelatif D. et al. Global epidemiology of nonalcoholic fatty liver disease-Meta-analytic assessment of prevalence, incidence, and outcomes. Hepatology (Baltimore, MD). 2016;64(1):73-84.

2. Ley R.E., Turnbaugh P.J., Klein S., Gordon J.I. Microbial ecology: human gut microbes associated with obesity. Nature. 2006;444(7122):1022-1023.

3. HuttenhowerC., Gevers D., KnightR. etal.Structure, function and diversity of the healthy human microbiome. Nature. 2012;486(7402):207-214. https ://doi.org/10.1038/nature11234.

4. Gillespie J.J., Wattam A.R., Cammer S.A. et al.PATRIC: the comprehensive bacterial bioinformatics resource with a focus on human pathogenic species. Infect Immun. 2011;79(11):4286-4298.

5. Swidsinski A., Loening-Baucke V., Lochs H., Hale L.P. Spatial organization of bacterial fora in normal and infamed intestine: a fuorescence in situ hybridization study in mice. World J Gastroenterol.2005;11(8):1131-1140.

6. Brown C.T., Sharon I., Thomas B.C. et al. Genome resolved analysis of a premature infant gut microbial community reveals a Varibaculum cambriense genome and a shift towards fermentation-based metabolism during the third week of life. Microbiome. 2013;1(1):30. https ://doi.org/10.1186/2049-2618-1-30.

7. Clemente J.C., Ursell L.K., Parfrey L.W., Knight R. The impact of the gut microbiota on human health: an integrative view. Cell. 2012;148(6):1258-1270.

8. Macpherson A.J., de Aguero M.G., Ganal-Vonarburg S.C. How nutrition and the maternal microbiota shape the neonatal immune system. Nat Rev Immunol. 2017;17(8):508-517.

9. Kovacs A., Ben-Jacob N., Tayem H. et al. Genotype is a stronger determinant than sex of the mouse gut microbiota. Microb Ecol. 2011;61(2):423-428.

10. Turnbaugh P.J., Ridaura V.K., Faith J.J. et al. The efect of diet on the human gut microbiome: a metagenomic analysis in humanized gnotobiotic mice. Sci Transl Med. 2009;1(6):6-14. https://doi. org/10.1126/scitranslm ed.3000322.

11. Yatsunenko T., Rey F.E.,Manary M.J. et al. Human gut microbiome viewed across age and geography. Nature. 2012;486(7402):222-227.

12. Sleisenger M.H., Feldman M., Friedman L.S., Brandt L.J. Sleisenger and Fordtran's Gastrointestinal and Liver Disease: Pathophysiology, Diagnosis, Management, 9th ed. Philadelphia, PA: Saunders/Elsevier. 2010.

13. Drenick E.J., Fisler J., Johnson D. Hepatic steatosis after intestinal bypass-prevention and reversal by metronidazole, irrespective of protein-calorie malnutrition. Gastroenterology. 1982;82(3):535-548.

14. Mouzaki M., Comelli E.M., Arendt B.M. et al. Intestinal microbiota in patients with nonalcoholic fatty liver disease. Hepatology (Baltimore, MD). 2013;58(1):120-127.

15. Zhu L., Baker S.S., Gill C. et al. Characterization of gut microbiomes in nonalcoholic steatohepatitis (NASH) patients: a connection between endogenous alcohol and NASH. Hepatology (Baltimore, MD). 2013;57(2):601-609.

16. Yari Z., Rahimlou M., Eslamparast T. et al. Flaxseed supplementation in non-alcoholic fatty liver disease: a pilot randomized, open labeled, controlled study. Int J Food Sci Nutr. 2016;67(4):461-469.

17. Wang B., Jiang X., Cao M. et al. Altered fecal microbiota correlates with liver biochemistry in nonobese patients with non-alcoholic fatty liver disease. Sci Rep. 2016;6:32002.

18. Loomba R., SeguritanV., LiW. et al. Gut microbiome-based metagenomic signature for non-invasive detection of advanced fibrosis in human nonalcoholic fatty liver disease. Cell Metab. 2017;25(5):1054-1062. e1055. 
19. Wong V.W., Tse C.H., Lam T.T. et al. Molecular characterization of the fecal microbiota in patients with nonalcoholic steatohepatitis-a longitudinal study. PLoS One. 2013;8(4):e62885.

20. Sleisenger M.H., Feldman M., Friedman L.S., Brandt L.J. Sleisenger and Fordtran's Gastrointestinal and Liver Disease: Pathophysiology, Diagnosis, Management, 9th ed. Philadelphia, PA: Saunders/Elsevier. 2010.

21. David L.A., MauriceC.F., Carmody, R.N. et al. Diet rapidly and reproducibly alters the human gut microbiome. Nature. 2014;505:559-563. doi:10.1038/nature12820.

22. Muegge B.D., Kuczynski J., Knights D. et al. Diet drives convergence in gut microbiome functions across mammalian phylogeny and within humans. Science. 2011;332: 970-974. doi:10.1126/science.1198719.

23. ShankarV., Gouda M., Moncivaiz J. et al. Differences in gut metabolites and microbial composition and functions between Egyptian and U.S. children are consistent with their diets. mSystems. 2017;2: e00169-16.

24. Voigt R.M., Summa K.C., Forsyth C.B. et al. The circadian clock mutation promotes intestinal dysbiosis. Alcohol.-Clin. Exp. Res. 2016;40:335-347.

25. Dethlefsen L., Huse S., Sogin M.L., Relman D.A. The pervasive efects of an antibiotic on the human gut microbiota, as revealed by deep 165 rRNA sequencing. PLoS Biol. 2008;6(11):e280.

26. Madrid A.M., Hurtado C., Venegas M. et al. Long-Term treatment with cisapride and antibiotics in liver cirrhosis: efect on small intestinal motility, bacterial overgrowth, and liver function. Am J Gastroenterol. 2001;96(4):1251-1255.

27. Wu W. Small intestinal bacteria overgrowth decreases small intestinal motility in the NASH rats. World J. Gastroenterol. 2008;14: 313.

28. Dao M.C., Everard A., Aron-Wisnewsky J. et al. Akkermansia muciniphila and improved metabolic health during a dietary intervention in obesity: relationship with gut microbiome richness and ecology. Gut. 2016;65: 426-436. DOI 10.1136/gutjnl-2014-308778.
29. Ridaura V.K., Faith J.J., Rey F.E. et al. Gut microbiota from twins discordant for obesity modulate metabolism in mice. Science. 2013; 341 (6150): 1241214. DOI 10.1126/science.1241214.

30. Koeth R.A., Wang Z., Levison B.S. et al. Intestinal microbiota metabolism of L-carnitine, a nutrient in red meat, promotes atherosclerosis. Nat. Med. 2013;19:576-585. DOI 10.1038/nm.3145.

\section{ORCID and contributionship:}

Tetyana V. Koval: 0000-0001-8904-041X ${ }^{A, B, D}$

Ivan V. Chopey: 0000-0003-4626-0855 E,F

Mykhaylo M. Hechko: 0000-0003-2793-5044 A,B

Artur V. Kurakh 0000-0002-2763-2935 B,E

\section{Conflict of interest:}

The Authors declare no conflict of interest

\section{CORRESPONDING AUTHOR}

\section{Tetyana V. Koval}

Uzhhorod National University

77 Minayska St., 88000 Uzhhorod, Ukraine

tel: +3806664382879

e-mail: t.duxowi41992@gmail.com

Received: 05.11 .2020

Accepted: 02.03.2021

A - Work concept and design, B - Data collection and analysis, C - Responsibility for statistical analysis, D-Writing the article, $\mathbf{E}$-Critical review, $\mathbf{F}$ - Final approval of the article 